\title{
LA-UR?- $97-2761$
}

Approved for public release;

distribution is unlimited.

Title:

CASE STUDY: WILDFIRE VISUALIZATION

CONF-970744- -

Author(s):

James Ahrens, CIC-8/ACL

Patrick MCCormick, CIC-8/ACL

James Bossert, EES-8

Jon Reisner, EES-8

Judith Winterkamp, EES-8

Submitted to:

IEEE Visualization ' 97

Phoenix, AZ

RECENED

Nov 031997

OSTI

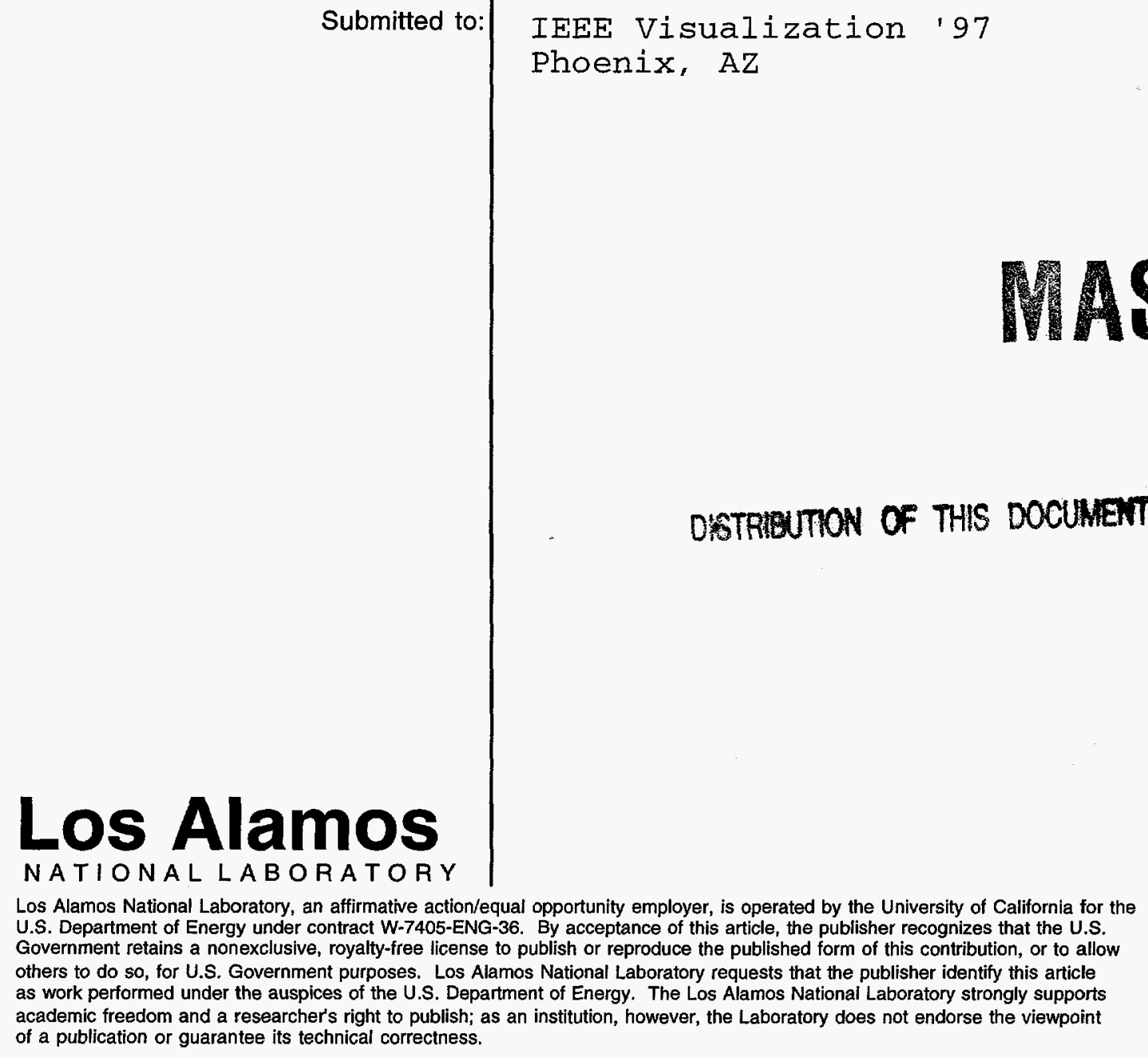

STER

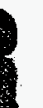

$\pm$

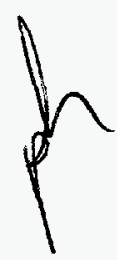
of a publication or guarantee its technical correctness. 


\section{DECLAMMER}

Portions of this doenment may be illegible in electronic image products. Images are produced from the best available original document. 


\section{DISCLAMER}

This report was prepared as an account of work sponsored by an agency of the United States Government. Neither the United States Government nor any agency thereof, nor any of their employees, makes any warranty, express or implied, or assumes any legal liability or responsibility for the accuracy, completeness, or usefulness of any information, apparatus, product, or process disclosed, or represents that its use would not infringe privately owned rights. Reference herein to any specific commercial product, process, or service by trade name, trademark, manufacturer, or otherwise does not necessarily constitute or imply its endorsement, recommendation, or favoring by the United States Government or any agency thereof. The views and opinions of authors expressed herein do not necessarily state or reflect those of the United States Government or any agency thereof. 


\section{Case Study: Wildfire Visualization}

\author{
James Ahrens, Patrick McCormick \\ Advanced Computing Laboratory
}

\author{
James Bossert, Jon Reisner and Judith Winterkamp \\ Earth and Environmental Sciences Division
}

Los Alamos National Laboratory

\begin{abstract}
The ability to forecast the progress of crisis events would significantly reduce human suffering and loss of life, the destruction of property, and expenditures for assessment and recovery. Los Alamos National Laboratory has established a scientific thrust in crisis forecasting to address this national challenge. In the initial phase of this project, scientists at Los Alamos are developing computer models to predict the spread of a wildfire. Visualization of the results of the wildfire simulation will be used by scientists to assess the quality of the simulation and eventually by fire personnel as a visual forecast of the wildfire's evolution. The fire personnel and scientists want the visualization to look as realistic as possible without compromising scientific accuracy. This paper describes how the visualization was created, analyzes the tools and approach that was used, and suggests directions for future work and research.
\end{abstract}

\section{Introduction to Wildfire Simulation}

Newspaper headlines constantly remind us of the human and property losses we suffer from wildfires, severe storms, earthquakes, and other disasters. These disasters cost the United States hundreds of lives and billions of dollars annually. Scientists at Los Alamos National Laboratory are developing computer models to predict the evolution of such disasters. By predicting the course of these events in faster than real-time, management strategies can be developed to minimize their adverse consequences. At present, the complexity of models that forecast crises require the advanced computing systems available at Los Alamos. In the near future, these models will be adapted for use in planning, training, and operational situations.

In the initial phase of this crisis forecasting project, scientists are developing computer models to predict the spread of a wildfire. These models will simulate both fire behavior and the local and regional weather conditions that affect a fire. To complement the modeling effort, Los Alamos researchers are partnering with Los Angeles County Fire Department, United States Forest Service, and Kennedy Space Center personnel who provide real-world wildfire expertise and fire measurements.

Fire behavior is highly dependent upon winds, temperatures, and moisture. It is crucial to predict these weather parameters over the small regions where they directly affect a fire. Weather conditions in these small regions are driven by dynamic weather patterns such as cold fronts, and high pressure systems that develop over much larger geographical areas. The Regional Atmospheric Modeling System (RAMS), originally developed at Colorado State University, is used to predict these variable weather patterns. The RAMS model uses measurements from weather stations all over the country to predict winds, temperatures, and moisture into the near future. RAMS then accurately translates this information to increasingly smaller geographical areas. This "microscoping" of information is critical in mountainous regions where local conditions can be greatly affected by the terrain.

Weather predictions from RAMS in the vicinity of a fire are used by the high resolution model for strong gradient applications (HIGRAD) to accurately simulate weather variables across the fireline $[8,6]$. To model the interactions between winds and fire, HIGRAD has been combined with a simple fire behavior model from the US Forest Service (BEHAVE). This combined modeling system (HIGRAD/BEHAVE) is the first step in predicting the actual progression and heat release of a wildfire. This system has been used to simulate several recent fires. The first simulation was of the South Canyon fire that occurred near Glenwood Springs, Colorado on July 6,1994 . The second simulation was of the Calabasas fire that occurred near Malibu, California on October 22, 1996.

\section{Approach}

When visualizing the wildfire simulation the scientists want the results to look as realistic as possible without compromising scientific accuracy. Realism is considered an important goal because the visualization will be used to study the evolution of previous fires, train personnel how to respond to a wildfire and eventually be used to generate a visual forecast of a new fire's evolution. Since the visualization results will be used by fire personnel it is considered extremely important to remove any significant differences between the actual appearance of the fire and the results of the visualization. In addition, a realistic visualization simplifies the scientist's job of visually comparing the results of the simulation to their ground truth data: actual fires which are recorded on videotape.

The data input to the visualization consists of simulation results and the topography of the terrain. The simulation produces a temperature volume for the region of interest and a grid of where the fire has burned. This grid and the $x$ and $y$ coordinates of the temperature volume are evenly spaced rectilinear grids with dimensions of 1280 meters by 1280 meters with a 10 meter resolution. The $z$ dimension of the volume is represented using 101 irregularly spaced terrainfollowing sigma-coordinates. In addition, because we are currently modeling these fires after the fact, we have video and photographs of the region before, during and after the wildfire.

The visualization is composed of the following visual elements:

- Smoke and fire volume renderings based on the temperature data. Volume rendering is ideal for modeling the fire and smoke because of its ability to directly represent objects with transparency and light-emiting properties. It is important to note that the simulation and the resulting visualization are not directly modeling flames or smoke; they are derived from the temperature data generated by the simulation. The scientists provide a range of values for both the smoke and fire and a color mapping for these range. For the fire, yellow is the hottest range ( 340 degrees Kelvin and above) followed by orange ( 327 to 340 degrees) and red ( 315 to 327 degrees). The smoke is in the range 300 to 325 degrees and is gray in color.

- A polygonal mesh of the topography. A polygonal mesh of the topographic scalar data is created by making a collection of 3D points using a rectilinear grid of $x, y$ coordinates and the topographic data as the $z$ coordinate.

- A texture map of the terrain. A texture map of the terrain of the South Canyon and Calabasas region is created. An artist 
uses frames from video footage and photographs of the terrain to create tiles which are replicated and composed into one image. Contrasts between tiles are blended away and the image is modified to reflect topographic features such as wind swept appearance of the mountain tops in the South Canyon terrain.

- A texture map of the burned terrain. A texture map of a burnt view of the terrain is also created by mapping the colors of the original terrain texture map to a darkened grayscale. In addition, random patches of the original texture map are introduced into the burned texture map to simulate small areas missed by the fire. At the start of the Colorado simulation the burned region is not empty. The initial burned region was estimated in the simulation as a large trapaziodal area. When viewed in the resulting simulation this burned region looked unnatural. To compensate for this, random points are chosen in the outer boundary of the specified region. These points are then dilated and used to erode the burned region. The burned region is then smoothed.

These elements are composed together to form an image. First, a composite texture map is created by using the simulation's burned region field as a mask to select texture either from the burned or original texture maps. The composite texture map is then mapped onto the polygonal mesh of the topography. The polygonal mesh is then rendered along with the smoke and fire volumes using a perspective projection in order to create the final image.

\section{Implementation}

Our target machine is a 32 processor SGI Origin 2000 with four Infinite Reality graphics pipes each with $64 \mathrm{MB}$ of texture memory. We had a time frame of about a month and a half to produce a program to produce the visualization video sequences for both simulations. Therefore we wanted to use an existing package that allowed us to quickly create the visualization. We choose to use IBM's Visualization Data Explorer (DX) product. Data Explorer provides a full collection of visualization tools and uses a data-flow network to represent programs. This allows for fast program prototyping and creation. DX provides the functionality to volume render the smoke and fire in software and texture map the terrain mesh in hardware. However, DX does not currently support the compositing of software and hardware rendering. An alternative method, using software texture mapping is not currently available in DX. Therefore we were forced to compute a composite result as part of the visualization. It was also difficult to create appropriate transfer functions for the volume renderer. DX uses a colormap as a transfer function and thus the colormap interface is the only interactive method for specifying transfer functions. In general we found that although a single tool, such as a standalone volume renderer might provide better functionality for a specific operation, it was invaluable to have a complete collection of tools which were designed to work together.

To achieve good performance, we used the Symmetric Multiprocessor (SMP) version of Data Explorer. This version exploits dataparallelism within modules in the network. That is, a module's data is partitioned to multiple processors and multiple threads process the data. Table 1 presents timing results for the wildfire network on various numbers of processors. The results show that DX can achieve a significant speedup using up to 8 processors; notice the speedup falls off with a greater number of processors.

\section{Results and Analysis}

Images generated by the visualization are shown in Figures 2 thorough 7 . Figures 2 through 4 shows images generated for the South
Table 1: DX Wildfire Network SMP timing results

\begin{tabular}{|l|l|l|l|l|l|l|}
\hline Procs. & 1 & 2 & 4 & 8 & 16 & 24 \\
\hline \hline Time in secs. & 204 & 120 & 67 & 44 & 32 & 27 \\
\hline
\end{tabular}

Canyon fire near Glenwood Springs, Colorado. Figures 5 through 7 shows images generated for the Calabasas fire near Malibu, California. An accompanying video shows the complete visualization sequences for both simulations. The simulation and resulting visualization accurately portray how the fire evolved in real-life. For example, in the Colorado fire, strong winds were gusting up the mountain tops (i.e. from lower left to upper right in Figures 2 through 4). These winds caused the fire, which had burned down the first mountain top slowly (i.e. the rightmost mountain top) to race up the valley and quickly cover and consume the second mountain top (i.e. the mountain top where the fire is burning in Figures 3 and 4). Tragically, fourteen firefighters were stationed on this mountain and lost their lives in the fire.

For the two studied simulations the scientists plan to analyze the results in a qualitative manner since the real-world data they are comparing with is video and summarized personal accounts of the fire's spatial and temporal behavior. A United States Government Interagency investigation of the South Canyon Fire was done in 1994 to study all aspects of the response to this fire. The report contains a figure which summarizes a set of personal accounts of the fire's behavior [5] (Figure 1). The report's accounts of the path taken by the fire (i.e. from $A$ to $H$ ), the overall time to burn from $A$ to $\mathrm{H}$ (i.e. 20 minutes) and the fast progress from $\mathrm{D}$ to $\mathrm{H}$, agree well with the simulation results for the South Canyon Fire.

In the future, the scientists will compare their results to the output of the AIRDAS system, an airborne scanning instrument designed to record information about wildfire[4]. The system includes detectors for fuel condition assessment and fire temperature measurements. The data generated from this system will allow the scientists to quantitatively assess the results of their simulation. Future work includes creating a new visualization algorithm which is similar in style to the output of the scanning instrument in order to facilitate the comparison process.

\section{Related Work}

Related work to wildfire visualization include research on visually convincing models of flame and smoke. Stam and Fiume describe models to represent flame, smoke and a fire's spread[9]. Their work differs from ours because we have an additional, possibly conflicting goal beyond creating a visually convincing model, which is to portray the results of the simulation as accurately as possible. Clark et. al. at the National Center for Atmospheric Research are also working on a coupled atmosphere/fire model[2]. Their published visualization results[1] of their simulations do not attempt to provide a visually accurate visualization. Other work in this area, includes work by Rushmeier et. al.[7] on using graphics techniques, such as ray casting in order to better understand the radiative effects of fires.

\section{Conclusions and Future Work}

We have created a realistic visualization for wildfire simulations using IBM's Data Explorer. In the future, we plan to extend the system to provide the results in faster than real-time in order to create and deliver a forecast of a wildfire's evolution. Some of the steps involved in this process are: 


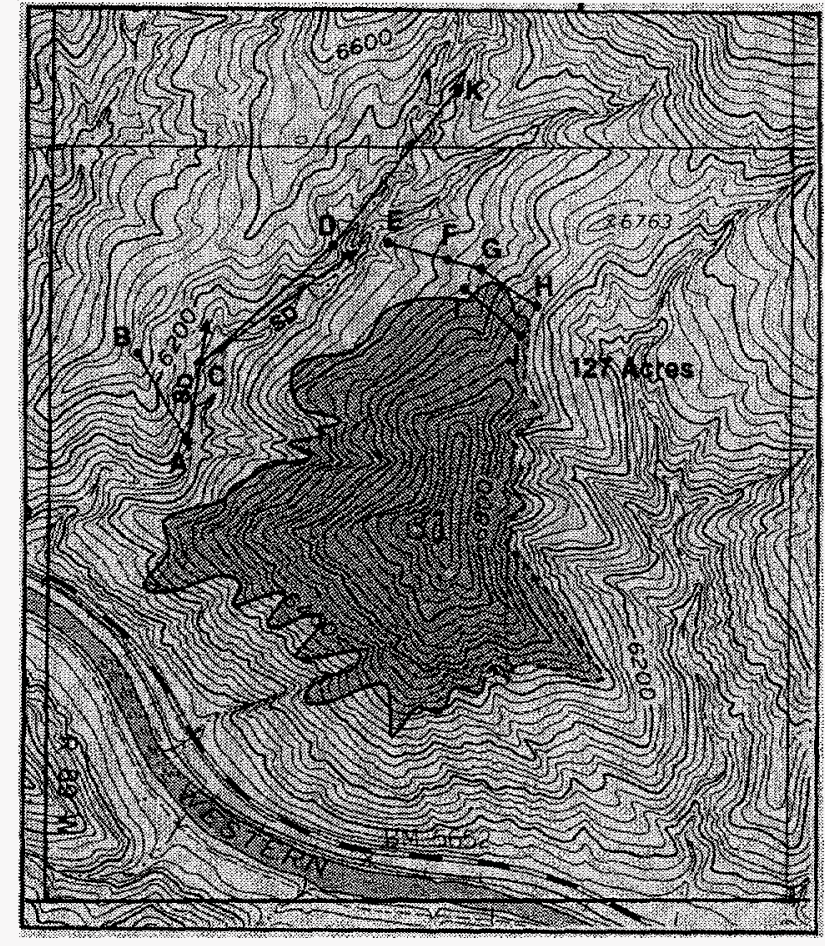

Figure 1: From the U.S. Government Interagency Report on the South Canyon Fire [5], Fire Behavior Calculations.

- Input Gathering - The topography, weather data and initial fire conditions need to be gathered extremely quickly. In addition, we would like to increase realism by visualizing actual terrain data obtained from aircraft or satellites. The REINAS system, a system for gathering environmental data in realtime, may be useful in this process [3].

- Simulation - The simulation currently runs slower than realtime. The scientists are porting their code to the Origin 2000 and improving its performance. Their goal is to obtain results at least 5 times faster than real-time in order for the simulation to be useful as a predictive tool.

- Visualization - Currently the visualization runs for $27 \mathrm{sec}-$ onds to generate 1 second of real-time data on 24 processors of the Origin 2000. The performance bottleneck of the visualization is the software volume rendering of the smoke and fire. One option for improving performance is to extend Data Explorer to support both hardware volume rendering and hardware texture mapping. An alternative is to create a custom scalable software-based parallel volume rendering module for DX.

- Delivery - The resulting visualization needs to be delivered to fire personnel at the fire's location. Radio and satellite communication equipment will most likely be used to communicate the results to laptop computers at the wildfire site.

\section{Acknowledgments}

We would like to thank the other members of the ACL Visualization Team, Jamie Painter and Michael Krogh for their support and advice. We would also like to thank the members of IBM Data Explorer Team, especially Greg Abram, Paula Austel, Nancy Collins and Lloyd Trenish whose advice and suggestions on Data Explorer have been key to the success of the project. Thanks to Genevieve Fox for creating the texture maps. We acknowledge the Advanced Computing Laboratory of Los Alamos National Laboratory, Los Alamos, NM 87545. This work was performed on computing resources located at this facility.

\section{References}

[1] T. Clark, J. Coen, and D. Middleton. A numerical model of an explosive forest fire - visualization gallery. National Center for Atmospheric Research. Available from http://www.scd.ucar.edu/vg/FIRE/ClarkFire.html.

[2] T. Clark, M. Jenkins, J. Coen, and D. Packham. A coupled atmosphere-fire model: Convective feedback on fire line dynamics. Joumal of Applied Meteorology, 35(6):875-901, 1996.

[3] D. Long et. al. REINAS: A real-time system for managing environmental data. In Conference on Software Engineering and Knowledge Engineering, June 1996.

[4] J. Brass et, al. Airborne infrared disaster assessment system (AIRDAS). NASA Ames Research Center. Available from http://geo.arc.nasa.gov/sge/brass/Brass.AIRDAS.html.

[5] Roskenkrance et. al. Interagency report on the south canyon fire-accident investigation team. Bureau of Land Management and USDA Forest Service, August 1994.

[6] J. Reisner and P. Smolarkiewicz. Thermally forced low froude number flow past three-dimensional obstacles. Journal of Atmospheric Sciences, 51(1):117-33, 1994.

[7] H. Rushmeier, A. Hamins, and M. Y. Choi. Volume rendering of pool fire data. IEEE Computer Graphics and Applications, 15(4):62-7, 1995.

[8] P. Smolarkiewicz and L. Margolin. On forward-in-time differencing for fluids: An eulerian/semi-lagrangian nonhydrostatic model for stratified flows. Atmospheres and Oceans, in press, 1996.

[9] Jos Stam and Eugene Fiume. Depicting fire and other gaseous phenomena using diffusion processes. In Robert Cook, editor, SIGGRAPH 95 Conference Proceedings, Annual Conference Series, pages 129-136. ACM SIGGRAPH, August 1995. 


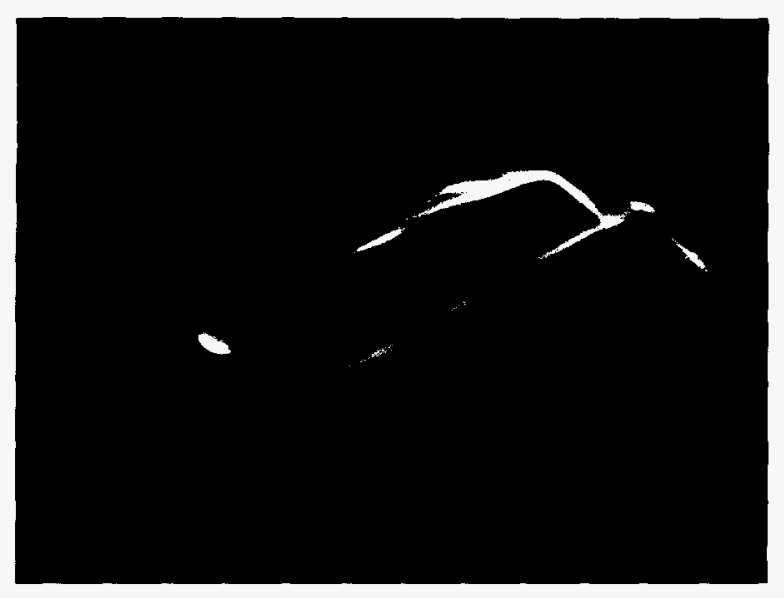

Figure 2: Image from the July 6, 1994, South Canyon fire near Glenwood Springs, Colorado at the start of the simulation.

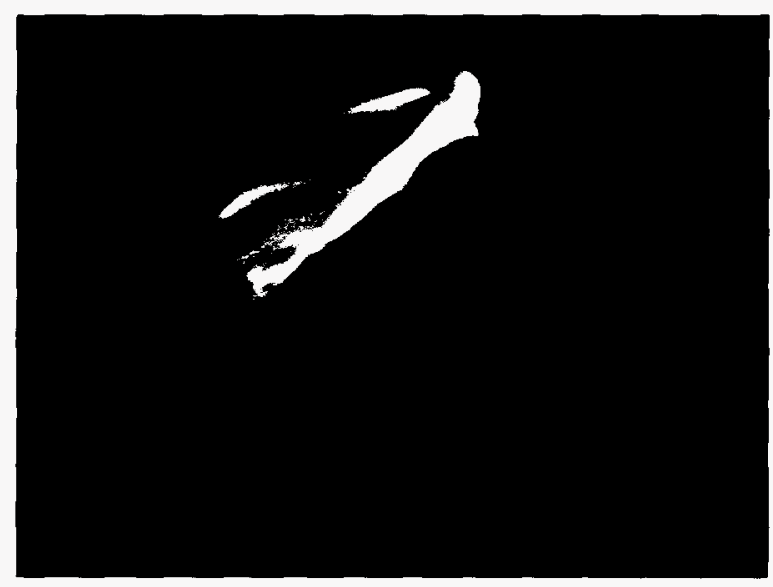

Figure 3: Image from 5 minutes into the simulation.

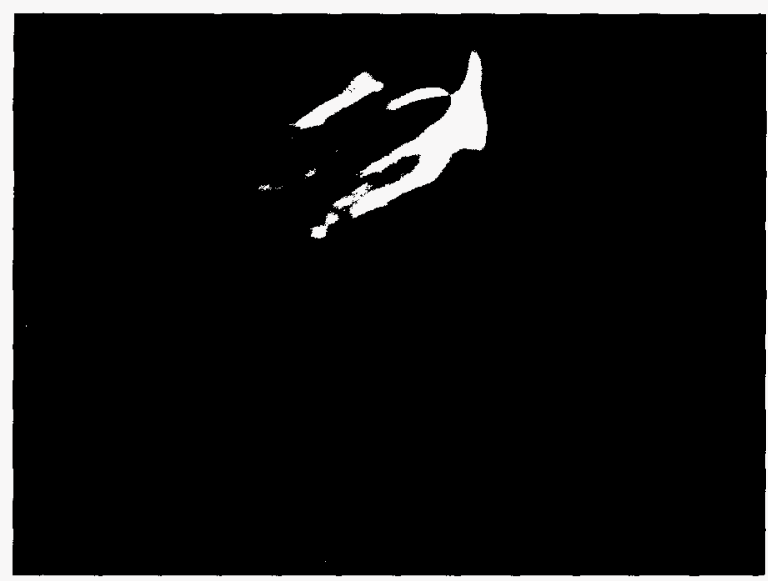

Figure 4: Image from 10 minutes into the simulation.

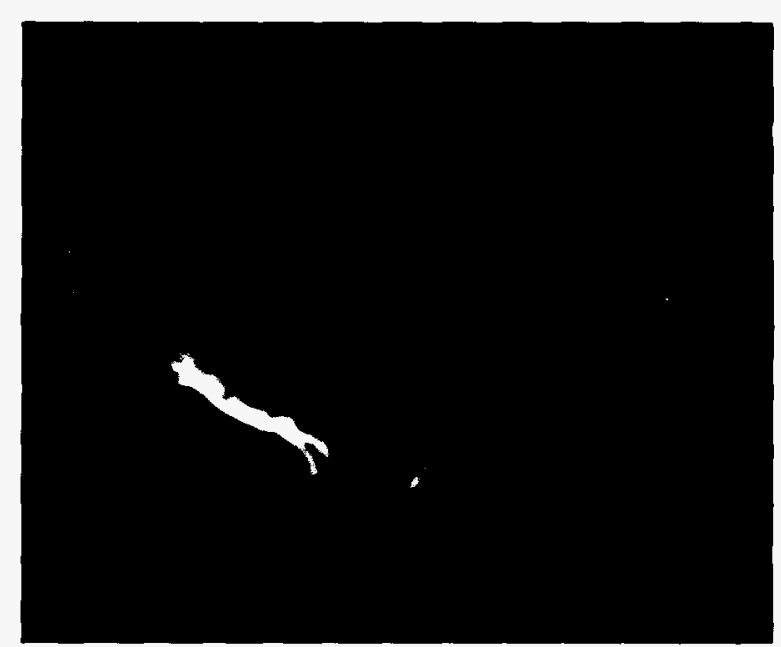

Figure 5: Image from the October 22, 1996, Calabasas fire near Malibu, California, at 33.5 minutes into the simulation.

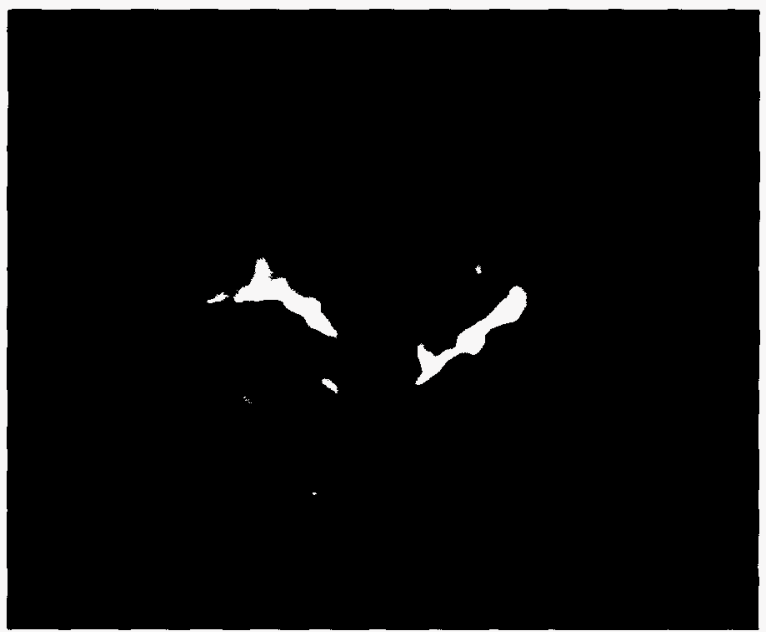

Figure 6: Image from 52 minutes into the simulation.

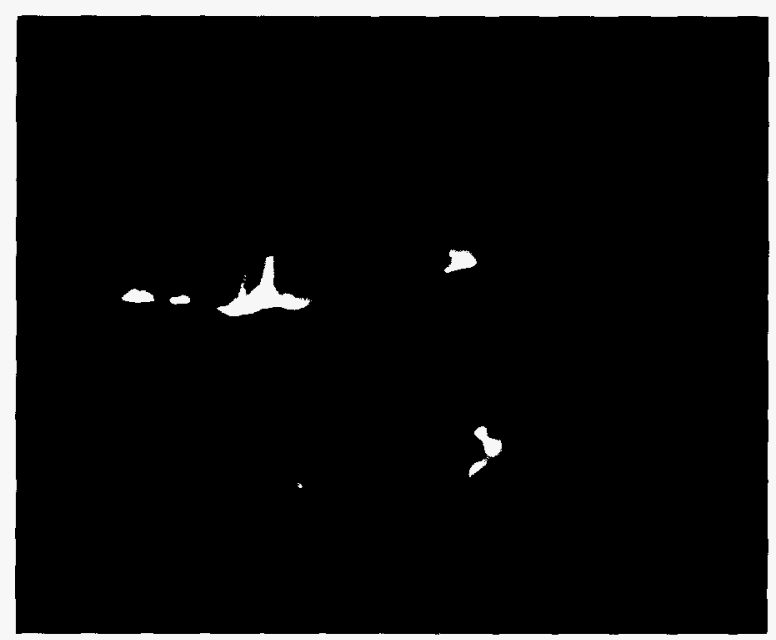

Figure 7: Image from 68.5 minutes into the simulation. 